\section{$\underset{\text { hommes }}{\text { \& migrations }}$}

\section{Hommes \& migrations}

Revue française de référence sur les dynamiques

migratoires

1305 | 2014

L'exil chilien en France

\title{
Le poids de la défaite
}

Retour sur les origines de l'exil politique chilien (1970-1990)

\section{Franck Gaudichaud}

\section{(2) OpenEdition}

1 Journals

\section{Édition électronique}

URL : http://journals.openedition.org/hommesmigrations/2707

DOI : 10.4000/hommesmigrations.2707

ISSN : 2262-3353

Éditeur

Musée national de l'histoire de l'immigration

\section{Édition imprimée}

Date de publication : 1 janvier 2014

Pagination : 9-15

ISBN : 978-2919-040261

ISSN : 1142-852X

\section{Référence électronique}

Franck Gaudichaud, «Le poids de la défaite », Hommes \& migrations [En ligne], 1305 | 2014, mis en ligne le 01 janvier 2017, consulté le 19 avril 2019. URL : http://journals.openedition.org/

hommesmigrations/2707 ; DOI : 10.4000/hommesmigrations.2707 


\title{
LE POIDS DE LA DÉFAITE RETOUR SUR LES ORIGINES DE L'EXIL POLITIQUE CHILIEN (1970-1990)
}

par FRANCK GAUDICHAUD, docteur en science politique et maître de conférences en civilisation de l'Amérique latine à l'université de Grenoble, spécialiste du Chili, chercheur de l'ILCEA (E.A.613).

\author{
La prise de pouvoir sanglante du général Pinochet, \\ en septembre 1973, a conduit à l'exil des centaines de milliers \\ de Chiliens, parmi eux, les militants de l'Unité populaire, \\ coalition de gauche dont la tête de file était le président \\ Salvador Allende. La dictature militaire tentera d'expurger, \\ à tous les étages de la société, la mémoire de cette "voie \\ chilienne au socialisme" tout en institutionnalisant la \\ répression et la traque des opposants. De nombreux réfugiés \\ n'auront alors de cesse de dénoncer le régime militaire, \\ de soutenir les actions de résistance sur place et de mobiliser la \\ solidarité internationale.
}

À quarante ans du coup d'État de septembre 1973, les images de La Moneda (le palais présidentiel) en flammes, ou celles du regard terrifié des prisonniers du stade national à Santiago du Chili restent encore gravées dans la mémoire de nombreux citoyens européens. Le peuple chilien, ses luttes et ses espoirs de transformation révolutionnaire ont été "au cœur" de toute une génération. C'est le cas en France notamment. Aujourd'hui, cette mémoire des résistances, de l'exil politique, du combat pour la défense des droits humains continue à marquer nos représentations collectives de ce petit pays du Cône sud du continent américain. Bien entendu, cette mémoire a connu des altérations et diverses "irruptions" au gré des décennies. Par-delà les frontières, cette vaste frange de terre coincée entre Pacifique et cordillère des Andes, "monde du bout du monde” conté par lécrivain Luis Sepúlveda, éclaire par son histoire récente les grands conflits du siècle passé.

Le Chili a été un pays "laboratoire" et un pays "modèle1". Après avoir expérimenté une tentative de transition démocratique vers le socialisme, il a connu la violente mise en place d'une dictature militaire, anticipant l'avènement d'une nouvelle raison du monde, celle du néolibéralisme². Nous tenterons de résumer brièvement certaines des grandes étapes qui ont ponctué ce temps chilien en 
prise avec les tourbillons du "court XX siècle $^{3 \text { " et de }}$ rappeler comment la contre-révolution qui débute en 1973 est à l'origine de l'une des plus importantes vagues d'exil politique sud-américain.

\section{Le Chili en 1970 : un contexte prérévolutionnaire}

En 1970, les Chiliens sont à peine 9 millions. Pays du capitalisme minier, le Chili possède d'immenses richesses naturelles, dont la plus grande réserve mondiale de cuivre, majoritairement aux mains de capitaux étasuniens. Cette économie d'enclaves signifie aussi la dépendance par rapport au marché mondial et d'immenses inégalités sociales. Sur le plan politique, la patrie des grands poètes Pablo Neruda et Gabriela Mistral a la réputation de profiter d'une certaine stabilité institutionnelle, en comparaison avec ses voisins sud-américains. En témoigne d'ailleurs l'absence de changement de Constitution sur de longues périodes ${ }^{4}$. L'État chilien est un État fort, centraliste, autour duquel a su se fédérer une classe dominante soudée, tout en permettant l'intégration partielle de couches
Après avoir expérimenté une tentative de transition démocratique vers le socialisme, il a connu la violente mise en place d'une dictature militaire, anticipant l'avènement d'une nouvelle raison du monde, celle du néolibéralisme. sociales subalternes et certains progrès sociaux, cela non sans de multiples répressions, à commencer par celle du peuple indigène mapuche.

Depuis la création des sociétés de résistance (la Centrale unitaire des travailleurs), le mouvement ouvrier est un acteur historique essentiel. Il s'organise politiquement autour de deux grandes organisations : le Parti communiste (PCC), fondé en 1922, l'un des plus importants d'Amérique latine, et le Parti socialiste (PS), parti-mouvement où l'on trouve au coude à coude réformistes, guévaristes, trotskistes, etc. Ces deux partis s'inscrivent dans une longue tradition de luttes sociales, mais aussi de participation parlementaire et d'expérience gouvernementale (avec, dans les années 1930, la création de Fronts populaires). À la fin des années 1960, le projet de "révolution en liberté" du gouvernement démocrate-chrétien (de 1964 à 1970), soutenu activement par l'administration Kennedy, échoue. La croissance industrielle promise n'arrive pas. Les étudiants, la petite paysannerie, tout comme les pauvres de la ville (les "pobladores"), réclament des changements radicaux. La décomposition de la gestion populiste démocratechrétienne (DC) ouvre la voie à la gauche. Le leader socialiste Salvador Allende, quatre fois candidat à la présidence, ancien ministre, prend la tête de l'Unité populaire (UP). Cette coalition est portée par le PCC et le PS, mais aussi par des secteurs significatifs de la gauche chrétienne.

\section{Allende et la "voie chilienne au socialisme" : les espoirs d'un peuple}

Dans un contexte de fortes tensions sociales et de mobilisations collectives croissantes, Allende remporte l'élection de septembre 1970, avec seulement 36,6\% des voix (élection à un tour) face au candidat DC et à celui de la droite conservatrice. Cette victoire sur le fil traduit aussi l'irruption sur la scène politique de secteurs populaires qui en étaient jusque-là largement exclus. Les espoirs sont immenses, les difficultés aussi. En pleine guerre froide, Allende cherche à incarner une troisième voie. À la différence de l'expérience cubaine, il fait le pari stratégique que la révolution chilienne sera pacifique et légaliste. Cette "voie chilienne" doit être conduite de manière graduelle, institutionnelle, en respectant la Constitution libérale de 1925. L'idée force était que l'État serait suffisamment "flexible" et, condition sine qua non, que les forces armées respecteraient le nouveau gouvernement démocratique ${ }^{5}$. 
pales d'avril 1971. Cette politique menace directement les intérêts de l'oligarchie nationale, en plus d'être durement combattue par les États-Unis. La droite chilienne, avec l'appui des services secrets de Washington, se fixe pour objectif de désarticuler le bloc politique et social qui appuie le gouvernement ${ }^{6}$. Les attentats d'extrême droite se multiplient, une pression constante est exercée sur le Parti démocrate-chrétien (DC) afin qu'il bascule dans une opposition plus décidée et le grand patronat initie une tactique de boycott de l'économie. Les médias conservateurs (tel le quotidien El Mercurio), rouages essentiels de ce dispositif, font pression sur les forces armées afin qu'elles prennent position contre Allende face au "danger marxiste". Cette spirale se referme peu à peu sur le gouvernement ${ }^{7}$. Minoritaire au Parlement, enfermée dans des négociations parlementaires sans fin avec la DC, l'Unité populaire perd toute efficacité même sur le plan sur lequel elle avait décidé d'agir : celui des réformes structurelles impulsées depuis l'État.

Les "40 mesures" du programme de l'UP ont pour objectif de favoriser un développement économique soutenu, une politique audacieuse de redistribution des richesses, l'approfondissement de la réforme agraire et le contrôle des principales ressources nationales. L'expropriation du cuivre aux mains du capital étranger, la nationalisation de 91 grandes entreprises "monopolistiques" et des principales banques, permet la constitution d'une "aire de propriété sociale” (le secteur privé reste cependant largement majoritaire). Processus national et démocratique, le pays vit un véritable climat révolutionnaire dans différents champs sociaux : les grèves, les occupations de terres ou d'usines se multiplient. L'explosion de la participation collective favorise la gauche : l'Unité populaire capte environ $49 \%$ des voix lors des élections munici-

\section{L'issue tragique de la "bataille du Chili"}

Dans une telle conjoncture, la coalition de gauche s'est rapidement divisée entre un pôle modéré, réformiste, mené par les communistes et Allende, et un pôle partisan de la rupture, conduit par un secteur du PS, des chrétiens de gauche et soutenu par des organisations extra-parlementaires tel le Mouvement de la gauche révolutionnaire (MIR). Ces militants appellent à "avancer sans transiger" et à s'appuyer davantage sur le mouvement populaire organisé pour radicaliser le processus. Parallèlement, la polarisation politique s'invite dans la rue, les quartiers populaires, les universités et 
les industries. Le gouvernement lui-même semble dépassé par l'ampleur du conflit social. En réponse à chaque tentative séditieuse de la droite ou de "grève patronale", les formes d'auto-organisation, de ravitaillement direct, de contrôle ouvrier se multiplient (octobre 1972, juin 1973) : le "pouvoir populaire" devient, de manière transitoire, réalité et de nouvelles organisations apparaissent, tels les cordons industriels dans les ceintures des principales villes ${ }^{8}$.

Dépourvu d'une direction unifiée, persuadé du rôle "constitutionaliste" des militaires, le gouvernement espère jusqu'au bout pouvoir éviter la guerre civile. Le 11 septembre 1973, avec l'appui explicite de l'administration Nixon, un quarteron d'officiers organise le putsch. Parmi eux, un certain Augusto Pinochet, nommé par Allende quelques semaines auparavant chef des forces armées... La gauche se retrouve désarmée pour organiser la résistance. Au lieu de se rendre aux généraux félons, Allende se suicide dans un palais présidentiel bombardé ${ }^{9}$ La "bataille du Chili", admirablement filmée par Patricio Guzman en $1974^{10}$, vient de prendre dramatiquement fin. La défaite est profonde et durable : elle a des conséquences à long terme, qui se prolongent jusquà nos jours.

\section{Les années noires de la répression}

Le général Pinochet est un militaire catholique, anticommuniste et profondément conservateur. Il a dirigé d'une main de fer le régime militaire pendant presque dix-sept ans. S'appuyant sur l'idéologie de la "récupération nationale intégrale" et soutenu par la doctrine de la "sécurité nationale" propagée par les États-Unis, la dictature met en œuvre une politique de violation généralisée des droits humains.
Le 11 septembre, l'état de siège est déclaré par le décret-loi (DL) n ${ }^{\circ} 3$. Le 12, le DL $n^{\circ} 5$ confirme que l'état de siège doit être compris comme "État en temps de guerre" : toute personne pratiquant une forme quelconque de résistance sera "fusillée sur-le-champ". Les conseils de guerre sont immédiatement convoqués et pratiquent une "justice militaire" qui fonctionne de manière arbitraire et complètement autonome de la Cour suprême. L'état de siège est maintenu jusqu'en 1978 et rétabli par la suite, à deux reprises, dans les années 1980. La vie quotidienne, les actes les plus infimes, sont désormais rythmés par la peur, la délation et la répression. Contre le "cancer marxiste" qu'il s'agit d"extirper" du corps social, le terrorisme d'État s'abat sur le pays. Plus de 40000 victimes $^{11}$ sont désormais officiellement reconnues, suite à différentes commissions de "vérité et réconciliation". Les forces armées et la police politique ont torturé des dizaines de milliers de personnes, assassiné environ 3200 individus. Parmi eux, plus d'un millier sont des "détenus-disparus", dont les corps n'ont jamais été retrouvés ${ }^{12}$. Alors que les premiers mois sont ceux d'une brutalité aveugle, c'est un système d'information et de répression plus "sophistiqué" et sélectif qui se met par la suite en place, au travers de la Dina (Direction d'intelligence nationale) dirigée par Manuel Contreras. Les militants de gauche, syndicalistes, défenseurs des droits de l'homme, travailleurs sociaux, étudiants sont traqués, emprisonnés dans des camps ou des centres clandestins.

\section{L'institutionnalisation de la dictature}

Sur le plan institutionnel, les forces armées et la junte concentrent tous les pouvoirs, alors que sont détruits un à un les espaces sociaux et politiques 
démocratiques. L'ensemble des libertés civiles est mis à mal. Le 14 septembre 1973, le Congrès national est fermé manu militari et les mandats des parlementaires ou des maires sont annulés. Les partis politiques sont interdits. Les principales entreprises stratégiques du pays, en particulier les entreprises nationalisées sous Allende, sont immédiatement rendues à leurs ex-propriétaires. La censure s'abat sur la presse et la culture, les autodafés de livres jugés "subversifs" se multiplient. La classe ouvrière et les dirigeants syndicaux sont parmi les principales cibles des militaires. En 1975, les services publics sont réorganisés et des milliers de fonctionnaires sont mis sur liste noire : entre septembre 1973 et décembre 1975, environ 85000 salariés sont licenciés pour des raisons politiques (selon les chiffres officiels du Programme de reconnaissance des exonérés politiques du ministère de l'Intérieur).

Dans un souci de légitimation, le général Pinochet est déclaré "chef suprême de la nation" en 1974 et, après un plébiscite réalisé dans un contexte de peur et de censure, une nouvelle Constitution est promulguée en 1980. Dès 1978, le régime décrète une loi d'amnistie qui érige l'impunité pour tous les crimes commis. Dans sa déclaration de principes, la Constitution définit la dictature comme une "démocratie protégée" assurée par un "gouvernement apolitique, autoritaire et juste”. Par-delà une illusoire séparation des pouvoirs, ce sont le général Pinochet et le Conseil national de sécurité qui concentrent toutes les attributions. Le Sénat est en partie composé de membres nommés à vie et les maires désignés. La justice, la Cour suprême et les universités sont mises sous tutelle militaire $^{13}$. Une fois les syndicats "épurés", leurs dirigeants dépendent de la bonne volonté de la junte. Le général Pinochet manœuvre également pour écarter tous les généraux qui pourraient lui faire de l'ombre et s'entoure de nombreux civils (tel le juriste Jaime Guzmán), qui collaborent activement à la dictature.

\section{Exil et asile ${ }^{14}$}

Dans de telles conditions de terrorisme d'État, et malgré les consignes de certains partis comme le MIR de rester au Chili pour résister, des centaines de milliers de Chiliens sont contraints à l'exil (voir les articles à ce sujet dans ce numéro). Face à l'ampleur de la violence, qu'aucune organisation de gauche n'avait imaginée, nombre de dirigeants et militants de l'UP, parfois accompagnés de leurs familles, cherchent à fuir le pays. Plusieurs représentations diplomatiques et ambassades accueillent ceux qui parviennent à se réfugier dans leurs locaux, souvent au péril de leur vie et en bravant le couvre-feu. Les représentations vénézuéliennes ou mexicaines et suédoises, italiennes et françaises à Santiago se montrent particulièrement actives au moment de faciliter le départ de ces exilés
Face à l'ampleur de la violence, qu'aucune organisation de gauche n'avait imaginée, nombre de dirigeants et militants de I'UP, parfois accompagnés de leurs familles, cherchent à fuir le pays. politiques. Les réfugiés attendent souvent de longues semaines un sauf-conduit pour pouvoir, enfin, s'envoler vers un pays d'accueil, avec l'espoir (vite déçu) que cet exil ne sera que temporaire.

Dans cette bataille diplomatique, notons le rôle de Roland Husson, arrivé à Santiago en juin 1973 comme attaché culturel de l'ambassade de France. Sur la recommandation de l'ambassadeur Pierre de Menthon, un diplomate catholique choqué par la violence du coup d'État, il va aider à sauver des dizaines de vie. M. et Mme de Menthon offrent leur résidence et les trois étages de l'ambassade pour organiser l'accueil. Roland Husson se souvient : 'L'ambassadeur m'a demandé de filtrer et de recevoir ces gens et de lui donner des informations sur ce qu'ils voulaient. J'ai fait cela pendant trois mois. Dans le couloir de l'ambassade, huit ou dix jours après le coup d'État, il y avait entre une 
dizaine et une quinzaine de lits, des gens qui dormaient sur des lits de camp, soit des couples, soit des familles entières. Pendant dix ou quinze jours cela a été une sorte de camp pour les réfugiés ${ }^{15}$." Nombreux sont les exilés qui partent pour la France de cette manière. C'est le cas en particulier d'artistes chiliens, comme les acteurs Elsa Poblete ou Oscar Castro (actuel directeur du théâtre Aleph à Paris). L'Argentin Roberto Kosack, responsable jusqu'en 1979 des opérations de l'Organisation internationale des migrations (OIM), a lui aussi joué un rôle clé en aidant des milliers de personnes à sortir du pays, ce qui lui a valu en 1994 l'ordre du mérite Bernard O’Higgins (attribué par le gouvernement chilien) ${ }^{16}$. Plusieurs organismes humanitaires chi-

Les exilés politiques

deviennent rapidement les acteurs qui organisent cette solidarité depuis l'étranger. Ils sont d'ailleurs régulièrement qualifiés par la presse du régime de "traitres à la patrie" et d'"anti-Chiliens". liens et internationaux ont aussi contribué à l'organisation de ces départs.

De son côté, la junte met en place un arsenal juridique qui renforce cette vague d'exil forcé. Le décret-loi $n^{\circ} 81$, publié par le Journal officiel le 6 novembre 1973, détermine une liste de noms d'opposants qui ne sont plus autorisés à vivre au Chili. Commence alors à apparaître la figure répressive de l'extrañamiento - ou bannissement -, utilisée contre des milliers d'opposants. Par la suite, en août 1974, un nouveau décret-loi établit une liste de personnes interdites de séjour sur le territoire national ${ }^{17}$. Parmi les causes invoquées, la participation "à des actes contraires aux intérêts chiliens" ou encore la divulguation de certaines "doctrines" ou "idéologies", à commencer par le marxismeléninisme. Tous ceux et celles qui ne respecteraient pas cette consigne sont menacés de la peine de mort. Enfin, un décret d'avril 1975 propose à certains prisonniers politiques condamnés par les tribunaux militaires de voir leur peine commuée en bannissement, avec interdiction de retour.

\section{Solidarité internationale et organisation des exilés}

Les premières condamnations internationales de la dictature venant de l'ONU datent de 1974. L'Organisation des États américains (OEA) envoie une délégation en juillet 1974, qui visite 7 camps et lieux de détention, dont Chacabuco et Tejas Verde. En 1979, la commission des droits de l'Homme de l'ONU nomme un rapporteur spécial pour analyser la situation chilienne, ce qui provoque un grand mécontentement au sein de la junte. À l'étranger, la solidarité jaillit de multiples collectifs, associations, organisations religieuses et partis politiques de gauche. Des organismes tels qu'Amnesty International ou le tribunal Russel entendent aussi faire monter la pression contre Pinochet. Outre les manifestations, les réunions publiques, le soutien financier aux organisations de résistance, l'accueil des refugiés politiques apparaît comme l'un des "répertoires d'action solidaire" de cette époque. Les exilés politiques deviennent rapidement les acteurs qui organisent cette solidarité depuis l'étranger. Ils sont d'ailleurs régulièrement qualifiés par la presse du régime de "traîtres à la patrie" et d"'anti-Chiliens". Leur rôle a été essentiel dans la dénonciation permanente de la dictature et contre sa banalisation. Certaines figures de renommée internationale, tels Carlos Prats, ancien chef des forces armées et général "légaliste" sous Allende ; Orlando Letelier, ex-ministre des Affaires étrangères de l'UP ; Bernardo Leighton, dirigeant démocrate-chrétien qui s'est opposé au coup d'État (à la différence de la majorité de la direction de son parti), sont aussi perçues comme potentiellement dangereuses par le général Pinochet. Tous trois sont victimes d'attentats organisés par la Dina et ses alliés entre 1974 et 1976. Ces actions font partie de "l'Opération condor" ou, du moins, dans le cas de Prats, tout l'annonce : à partir de la fin 1975, 
la coordination croissante des dictatures du Cône sud se renforce pour combattre toutes les organisations révolutionnaires et pour détruire les résistances individuelles et collectives, y compris hors de l'Amérique latine, là où résident les exilés chiliens ${ }^{18}$.

\section{"Révolution" néolibérale et transition pactée}

Ces temps de brutalisation de la société sont aussi, dès 1975, ceux d'une thérapie de choc économique : la dictature forge une véritable "révolution capitaliste" en transformant le Chili en première expérimentation mondiale du néolibéralisme ${ }^{19}$. Ce changement radical a lieu sous les auspices d'universitaires de l'Université catholique (les "Chicago Boys"), formés aux préceptes de l'économiste étasunien Milton Friedman. Au cours d'une première étape de libéralisation accélérée (1973-1981), l'État privatise plus de 250 entreprises et banques, réduit impôts (moins 40 \%) et droits de douane. Cette politique permet de baisser l'inflation, et de doper la croissance tout en attirant investissements étrangers et spéculateurs avides de ressources : le mythe du "miracle chilien" est né, malgré la grande crise du début des années 1980. Certains chefs d'entreprise et des proches des militaires accumulent des fortunes, à commencer par le clan Pinochet. Le bilan social de cette curée est colossal. Le Code du travail est entièrement détricoté et des pans entiers des services publics marchandisés : destruction du système de retraites par répartition, municipalisation et privatisation de l'Éducation, retrait de l'État dans les transports, la santé, etc. Quand le dictateur abandonne le gouvernement, son pays est devenu l'un des plus inégalitaires du monde et la proportion de pauvres représente plus de $46 \%$ de la population (contre $28 \%$ en 1969). La fin du régime militaire est le produit d'une lente réactivation du mouvement social, de la réorganisation de l'opposition politique, de problèmes économiques croissants, mais aussi de la pression internationale. Les États-Unis ne soutiennent d'ailleurs plus cet allié embarrassant, pariant sur une relève plus "présentable". À partir de 1983, émergent d'importantes protestations populaires. Avec des hauts et des bas, le retour de l'état de siège n'arrive pas à empêcher révoltes et manifestations. L'Église catholique joue également un rôle marquant, grâce au cardinal Raul Silva Henríquez et au Vicariat de la solidarité du Chili qui accueillent les victimes de la dictature. Un secteur de l'opposition, organisé autour de la démocratie-chrétienne, décide alors de créer une "alliance démocratique" et commence à négocier avec la droite la sortie de la dictature. D'autres, comme le parti communiste ou le MIR, sont favorables à une stratégie de "rébellion populaire de masse" et même à une résistance politico-militaire. Cependant, plusieurs tentatives courageuses échouent ou sont désarticulées par la répression. C'est, par exemple, le cas de la guérilla du MIR à Neltume dans le sud du pays ou de l'attentat contre le général Pinochet en 1986 menée par le Front patriotique Manuel Rodriguez (FPMR). Suite à une légère ouverture du régime, la droite prépare une transition "pactée", avec l'accord des opposants les plus modérés : la démocratisation se fera, mais selon les règles dictées et contrôlées par la junte et ses alliés civils $^{20}$. Les années 1990 sont aussi le temps du retour pour de nombreux exilés, et souvent de la découverte de cet "autre Chili" forgé à feu et à sang: une "société néolibérale triomphante" s'est installée, où la violence de la modernité, de l'hyperconsommation et de l'individualisme a tout envahi ${ }^{21}$. Il faudra attendre les années 2000 pour voir ressurgir véritablement la mémoire de l'Unité populaire et le renouveau des mouvements sociaux, initiant ainsi une nouvelle période de l'histoire contemporaine chilienne. 